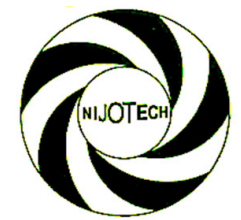

Nigerian Journal of Technology (NIJOTECH)

Vol. 35, No. 1, January 2016, pp. 25 - 30

Copyright(C) Faculty of Engineering, University of Nigeria, Nsukka,

Print ISSN: 0331-8443, Electronic ISSN: 2467-8821

www.nijotech.com

http://dx.doi.org/10.4314/njt.v35i1.4

\title{
MODIFIED TRAFFIC SIGNAL PHASING AT TRAFFIC WARDEN CONTROLLED INTERSECTION TO ACCOMMODATE PEDESTRIAN TRAFFIC IN ILORIN METROPOLIS, NIGERIA
}

\author{
0. O. Adeleke ${ }^{1 *}$, Y. A. Jimoh ${ }^{2}$ and I. T. Yusuf ${ }^{3}$ \\ 1, 2, 3 DePaRTMENT OF Civil ENGINEERING, UNIVERSITY OF ILORIN, ILORIN, KWARA STATE. NIGERIA. \\ E-mail addresses: ${ }^{1}$ adeleke.oo@unilorin.edu.ng, ${ }^{2}$ dryajimoh@yahoo.com, ${ }^{3}$ ityusuf@yahoo.com
}

\begin{abstract}
The study addresses the nervous anxiety always noticed in pedestrians as they cross at a 4-arm, Traffic Warden Control intersection in Ilorin metropolis, Nigeria. Two of the approaches (Challenge and Unity) are dualised multilanes while the other two (Obbo and CocaCola) are two-lane non-dualised. The present 4-phase traffic control scheme does not give consideration to pedestrian crossing. The geometric layout and the existing traffic management practice at the intersection were determined and a pedestrian traffic survey was conducted. The critical pedestrian volume of 171 pedestrians/hr was obtained on Challenge approach. Using an average walking speed of $1.2 \mathrm{~m} / \mathrm{s}$ and a crosswalk width of $3 \mathrm{~m}$ a pedestrian crossing time of 787.88 seconds within one hour is obtained as being adequate for the critical approach. A 5-phase scheme is proposed with the fifth phase being an all-stop exclusive phasing for pedestrian movements. Crosswalk should be introduced to concentrate pedestrian crossing activity at a specific location thereby positioning pedestrians to be more visible to traffic wardens and motorists.
\end{abstract}

Keywords: Traffic Warden Controlled Intersection, Traffic Conflict, Crosswalk, All-stop phasing

\section{INTRODUCTION}

1.1 Pedestrian Traffic

At Traffic Warden Controlled (TWC) intersections, vehicles are usually centered as priority with little or no regard to pedestrian traffic, putting them at conflict with vehicular traffic, especially on multilane movement corridors. Traffic warden controlled (TWC) intersection is prevalent in Ilorin metropolis, Nigeria, as in most other urban centres in the country [1]. The purpose of traffic control generally and TWC inclusive, is to assign the right-of-way to road users and thus facilitate safety by ensuring the orderly and predictable movement of all traffic on the roadways $[2,3]$. It is observed, however, that pedestrians are not given due consideration in the manual control (phasing) of traffic at congested TWC intersections unlike what is the case for intersections installed with traffic signal lights where phasing is at times designed to accommodate pedestrians and cyclists. This scenario makes crosswalk at TWC congested intersections to be disorderly, unpredictable and difficult, especially at high volumes, high speeds and multi lanes thereby making all pedestrians vulnerable; especially the aged and physically challenged [4]. A traffic signalization at TWC intersection designed to accommodate pedestrians will therefore not only enhance safety but also encourage walking as a mode of travel with additional benefits of enhanced health, reduction of vehicular traffic congestions and the emission of greenhouse gasses thereby creating a friendly environment $[5,6,7]$. Also, the old 'vehicle centred' notion in traffic engineering is now being replaced by the new 'human centred' notion which takes all road users into consideration in its planning, design and operations and attaches more importance to vulnerable traffic participants such as the pedestrians [4]. Thus the pedestrian traffic safety management at TWC intersections deserves a worthwhile re-examination.

Further, the recent push to increase non-motorized transportation in the last decade has led to an increased need for research and data relating to walking and walk ability $[8,9]$. The aim of the study is to design a modified traffic signal phasing at a TWC 
intersection to accommodate pedestrian traffic in order to improve safety and ease, for pedestrians crossing at high volume, congested, multilane TWC intersections. The focus is on the traffic management practice at a typical TWC intersection in Ilorin, an urban centre of a developing economy.

\subsection{Study Area}

The study area, Unity - Obbo-CocaCola road junction is a 4-arm at-grade, time-sharing intersection, situated in the Central Business District (CBD) of Ilorin metropolis, Nigeria. The approaches at the intersection are namely: Obbo Road, Coca Cola Road, Challenge and Taiwo Road. The intersection is controlled by traffic warden for about 14 hours, between $6: 30 \mathrm{am}$ to $8: 00 \mathrm{pm}$ everyday of the week. When not controlled, the intersection operates as a priority intersection with traffic on Obbo and CocaCola roads yielding to traffic on Taiwo and Challenge road approaches. All the approaches are paved with asphalt and the surfacing is in good condition. The pavements have no lane markings. Volumes of both vehicular and pedestrian traffics are high so also is the average vehicular speed. The pedestrian is presently not given any consideration in the phasing of traffic at the intersection which makes pedestrians to personally look for convenient acceptable gaps before crossing. Often times, nervous anxiety is seen in pedestrians at the intersection as they look for acceptable gaps to cross [10] or appeal to motorist to come to a halt so that they can safely cross the road. The indiscriminate appeal to motorists to come to a halt so that they can crosswalk, often leads to impedance of traffic flow on the approach sections. Sometimes, caring motorists also stop indiscriminately for pedestrians that are at the roadside intending to cross the roadway. All these actions lead to disorderly and unpredictable movement of pedestrian traffic leading to traffic delays, reduced level of service (LOS) and even bumper to rear vehicle accidents [11]. Adjoining the intersection are commercial buildings and a market which make the pedestrian traffic in the area high.

\subsection{Management of Pedestrian Traffic}

\subsubsection{Pedestrian crossing facilities:}

Many types of pedestrian crossings have been tried with varying degree of success, under two categories: (1) Uncontrolled Crossings and (2) Controlled Crossings. The typical example of the uncontrolled crossing is the zebra crossing. This consists of a sufficient width of crosswalk painted with black and white longitudinal strips so as to be conspicuous to the drivers as well as the pedestrians. As soon as a pedestrian steps onto the stripped crosswalk he has the right of way and all vehicles are required to stop legally. Apart from the stripped zebra markings in black and white, the crossing consists of a zigzag area (about 19 meters on both sides) to increase the drivers visibility of the crossing, black and white beacon posts, yellow flashing and studs to mark the limits of crossing[12]. Uncontrolled crossings are normally provided where the vehicular traffic is not heavy. If, however, the vehicular traffic is heavy, such crossing is likely to delay the vehicles to a great extent. In such cases signalised controlled crossing is considered. The simplest form of controlled crossing is one controlled by the police or traffic wardens. Other examples include traffic light signalized crossing with or without pedestrian push buttons. The signalized crossing with push button called the pelican crossing is operated by push button control when the waiting pedestrian demands a signal phase to cross. This is provided based on pedestrian and vehicular volumes. The inter-green period can also be used for pedestrian crossing. The studied intersection is controlled and has a high vehicular and pedestrian traffic volume thus a controlled pedestrian crossing is designed.

\subsubsection{Controlled pedestrian phasing overview:}

Some pedestrian phasing approaches include the following [13]:

1. Pedestrian movements served concurrently with the adjacent through movement phase at an intersection. This is done to simplify the operation of the intersection primarily. This approach of pedestrian operation has the possibility to put pedestrians in conflict with right-turning vehicles and left-turning vehicles that operate in a permissive mode, by inviting their movements at the same time [5].

2. Leading pedestrian interval where a leading pedestrian interval starts a few seconds before the adjacent through movement phase. This allows pedestrians to establish a presence in the crosswalk and thereby reduce conflicts with turning vehicles. This option supports improved safety for pedestrians by allowing them increased visibility within the intersection and is applicable to intersections where there are significant pedestrian-vehicle conflicts. 
3. Lagging pedestrian interval. A lagging pedestrian interval option operates similarly to a leading pedestrian interval, except that the pedestrian walk interval starts several seconds after the adjacent through movement phase. This option allows a waiting right-turn queue to clear before the pedestrian walk indication is presented and reduces conflicts with right-turning vehicles. It is applicable to intersections where there is: (i) a high right-turn volume and (ii) either an exclusive rightturn lane (or lanes) or the two intersecting roads have one-way traffic.

4. Exclusive pedestrian phase. An exclusive pedestrian phase dedicates an additional phase for the exclusive use of all pedestrians. This additional phase is configured such that no vehicular movements are served concurrently with pedestrian traffic. During this phase, pedestrians can cross any of the intersection legs and may even be allowed to cross the intersection in a diagonal path. This type of phasing has an advantage of reducing conflicts between right-turning vehicles and pedestrians, but it comes at a penalty of reduced vehicular capacity and longer cycle lengths (which increases delay to all users)[11, 14]. The exclusive pedestrian phase is not frequently used but can be found in the central business districts of several cities, including Denver and San Francisco in United States of America.

\section{MATERIALS AND METHODS}

Survey work was carried out using Total Station to map out the geometric layout of the intersection. Manual count was carried out to determine the pedestrian volume at the intersection. The 12-hour count was carried out from $7 \mathrm{am}-7 \mathrm{pm}$ in good weather condition for a week from Sunday to Saturday.

The existing traffic phasing at the intersection was determined through observatory method at morning peak period between $8.00-8: 30 \mathrm{am}$.
The various methods used to enhance pedestrian traffic movement at intersections were examined with a view to promoting traffic movements at the TWC intersection.

\section{RESULTS AND DISCUSSION}

\subsection{Intersection Geometric Layout}

Figure 1 and Table 1 present the layout of the intersection and the layout parameters respectively. Obbo road allows only one-way unidirectional southbound traffic flow. Although the width of Obbo road is $7.30 \mathrm{~m}$, traffic flow is constrained only to one lane as parked vehicles occupy the other lane space as a result of commercial activities adjacent to and along the road.

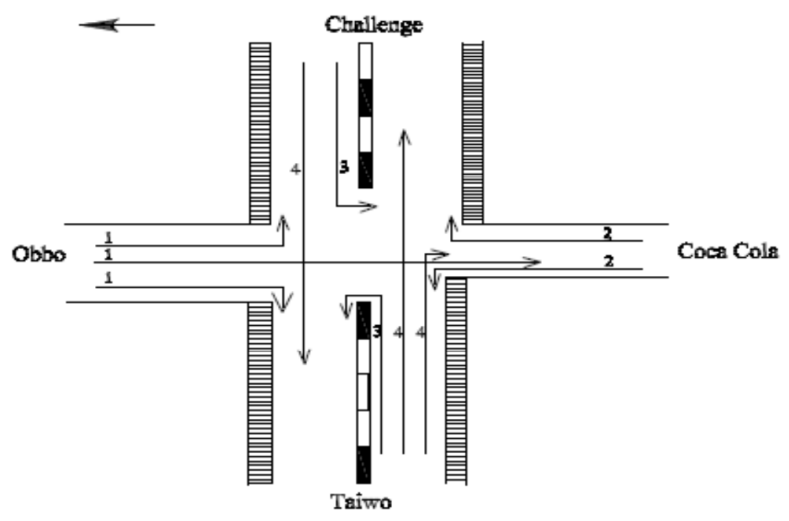

Fig. 1: Geometric Layout and Existing 4 Traffic Signal Phases of the Unity-Obbo-CocaCola Intersection

\subsection{Existing Traffic Management as Operated and Pedestrian Volume at the Intersection}

There is presently a four-traffic-signal-phasing operated by the traffic wardens at the studied intersection as depicted in Figure 1. A pedestrian survey was carried out for 12 hours each day from Sunday to Saturday to determine the pedestrian phasing time that will be adequate for pedestrian crossings. Table 2 shows the peak volumes at each of the approaches

Table 1: Inventory data for Unity-Coca Cola intersection

\begin{tabular}{ccccccc}
\hline Approach & $\begin{array}{c}\text { No of } \\
\text { approach } \\
\text { lanes in use }\end{array}$ & $\begin{array}{c}\text { Approach } \\
\text { width }(\mathrm{m})\end{array}$ & $\begin{array}{c}\text { Pedestrian } \\
\text { walkway(m) }\end{array}$ & $\begin{array}{c}\text { Opposing } \\
\text { lanewidth (m) }\end{array}$ & $\begin{array}{c}\text { Median } \\
\text { width (m) }\end{array}$ & $\begin{array}{c}\text { Overall road } \\
\text { width (m) }\end{array}$ \\
\hline Obbo & 1 & 10.60 & - & - & - & 10.60 \\
CocaCola & 1 & 3.65 & - & 3.65 & - & 7.30 \\
Taiwo & 2 & 9.40 & 2.5 & 10.60 & 1.50 & 21.50 \\
Challenge & 2 & 10.79 & 2.5 & 11.10 & 1.70 & 23.59 \\
\hline
\end{tabular}


Table 2: Peak hour pedestrian traffic volume

\begin{tabular}{ccc}
\hline Approach & Day and time & $\begin{array}{c}\text { Pedestrian volume } \\
\text { (ped/hr) }\end{array}$ \\
\hline Obbo & Fri, 6-7pm & 96 \\
CocaCola & Tue,11-12noon & 118 \\
Taiwo & Tue, 10-11am & 160 \\
Challenge & Sat,3-4pm & 171 \\
\hline
\end{tabular}

\subsection{Design of Pedestrian Safety Facility} 3.3.1 Pedestrian phasing

The various possible pedestrian traffic phasing approaches stated in Section 1.3.2 were examined in proposing a signal phasing that will accommodate pedestrian traffic at the intersection:

Approach No 1 where pedestrian movements are served concurrently with the adjacent through movement phase if operated

- for through movement from Obbo Rd (Phase 1), there is possible pedestrian movement conflict with right turning vehicles to Taiwo $\mathrm{Rd}$ and left turning vehicles to Challenge

- for through vehicular movements on Challenge and Taiwo approaches (Phase 4), there is possibility of conflict of pedestrians with right turning vehicles to Coca Cola Road.

Approaches No 2 and 3 - The fact that the intersection is manually controlled will make the leading and lagging pedestrian interval phasing type (approaches 2 and 3 above) difficult to apply as the traffic warden will find it difficult to give vehicle movement indication and shortly after give pedestrian movement indication. The several activities to be carried out almost simultaneously will make the operation complex for a manual controller, the Traffic Warden, to carry out efficiently.

Approach No 4- The all red option is considered most appropriate for the studied intersection as it will eliminate the possibility of vehicular/pedestrian traffic conflict. Crosswalk is suggested to be introduced at each approach of the intersection $[4,5]$. The Crosswalk will concentrate pedestrian crossing activity at a specific location and position pedestrians to be more visible by traffic warden/motorists.

\subsubsection{Determination of phasing time}

The signal phasing at TWC intersection is described as similar to what obtains in a vehicle actuated signalized intersection where the time allotted to an approach is a factor of how many vehicles are on the approaches waiting to cross [15]. The same could be applied in determining the time allotted for pedestrian crossing.
However there is the need to carry out an analysis on time budgeting at the intersection as a guide in sharing the cycle length.

In determining the sufficient phasing time required to accommodate pedestrian traffic, the pedestrian traffic volumes at the approaches were used to determine the critical crosswalk path which is defined as the approach with the highest volume of pedestrian traffic. In Table 2, the pedestrian volume on Challenge approach is critical which if taken care of allows pedestrians on the remaining approaches adequate time to cross. Equation 1 is used to determine the pedestrian crossing time (PCT). With a curb-curb width of $22.49 \mathrm{~m}$ and a walking speed of $1.22 \mathrm{~m} / \mathrm{s}$ [16], the PCT of $18.43 \mathrm{sec}$ is obtained for a pedestrian to cross the Challenge roadway which has the critical approach. If a crosswalk width of $3.0 \mathrm{~m}$ is provided which will allow 4 people walk side by side [17], for the critical pedestrian volume of 171 therefore a pedestrian phasing time (PHT) of $(18.43 \times 171) / 4$ $=787.88 \mathrm{sec}$ maximum will be desirable, in a period of $1 \mathrm{hr}$.

PCT $=W / V$

In (1), PCT is the pedestrian crossing time (sec), $\mathrm{W}$ is the width of roadway $(\mathrm{m}), \mathrm{V}$ is the pedestrian walking speed $(\mathrm{m} / \mathrm{s})$.

The existing 4-phase signalisation shown in Figure 1 is proposed to be modified to a 5-phase signalisation, the fifth phase being an all-stop phase for vehicular traffic to allow for enhanced pedestrian crossing [11]. If $787.88 \mathrm{sec}$ are required to adequately accommodate pedestrian traffic in one hour then the green time for the fifth phase is given by $(787.88 / \mathrm{n})$ sec where $\mathrm{n}$ is the number of cycles in a time period of one hour. In order to determine the number of cycles (n), the existing manual phasing pattern was observed for 30 minutes at a morning peak period and yielded the following phasing times: $6 \mathrm{sec}, 42 \mathrm{sec}, 10 \mathrm{sec}$ and 17 sec for phases $1,2,3$, and 4 respectively which give a cycle length of $75 \mathrm{sec}$. With a maximum time of 787.88 sec required in a period of 1 hour for the all-stop phase to accommodate pedestrian traffic the remaining period of (3600-787.88 $=2812.12)$ sec of the hour will be left for vehicular traffic. The value of $2812.12 \mathrm{sec}$ divided by the present cycle length of 75 sec yields $n$ i.e. 37 cycles in one hour. Therefore the pedestrian phasing time will be given by the required time of 787.88/37 which gives 21.29 sec or 22 sec. Thus the 5-phase signalisation that will be introduced at the intersection will have the following phasing 
times: Phase 1 - $6 \mathrm{sec}$, Phase 2 - $42 \mathrm{sec}$, Phase 3 - 10 sec, Phase $4-17$ sec and Phase 5 - 22 sec. Although the intersection is not a fixed time signalisation the value obtained for the green time of the fifth phase is computed to serve as guide.

\subsubsection{Design of crosswalk}

Each roadway should be provided with pedestrian bays and pedestrian crosswalk for pedestrians to aggregate and for crossings respectively. Stop lines are marked on each of the approaches to stop motor vehicles in advance of the marked crosswalks; this keeps the crosswalk clear for pedestrians and can reduce right-on-red conflicts. Figure 2 shows the proposed layout with Ladder crosswalk design that improves motorist vision and awareness.

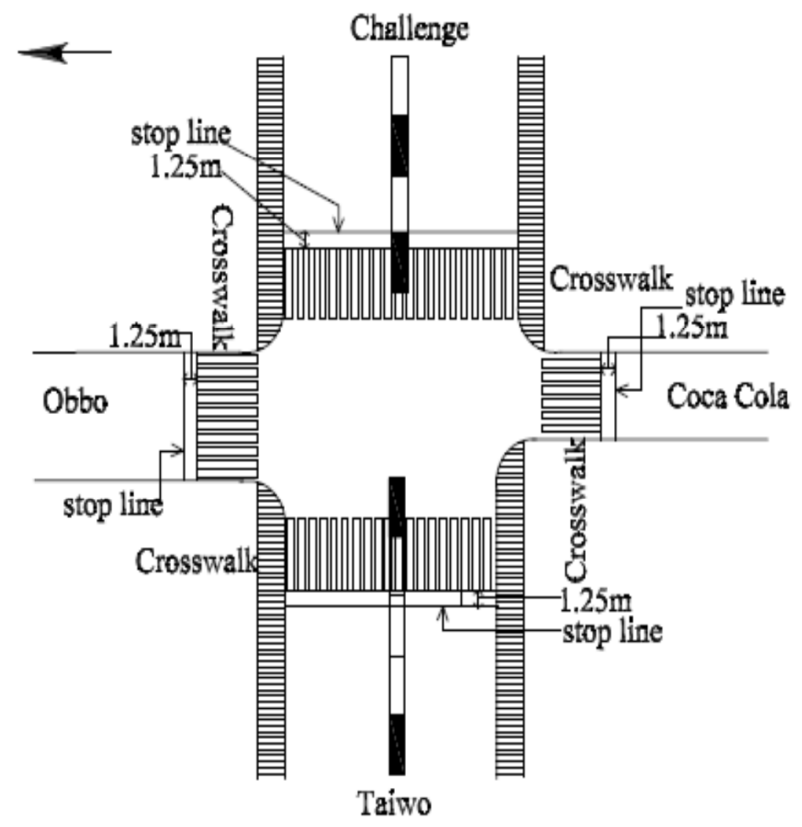

Fig. 2: Designed Crosswalk for the Unity-Obbo-CocaCola Intersection, Ilorin

\subsubsection{Pedestrian safety enhancement}

The present traffic situation at the intersection whereby pedestrians cross the road with nervous anxiety either waiting for acceptable gaps to cross or pleading to motorists to come to a halt so as to cross will be eliminated if the 5-phase signalization is introduced and well-coordinated by the Traffic Wardens. Orderly and predictable pedestrian crossing will replace the present disorderliness, the pedestrians especially the aged and physically challenged would also feel more secured crossing as risk of exposure to vehicular traffic conflict is minimized. This approach if introduced at TWC controlled intersections with multilane roads with high traffic volume at high speeds will not only enhance safety but also encourage walking as a mode of travel with the additional benefits of enhanced health, reduction of vehicular traffic congestions and the emission of green house gasses and thereby creating a friendly environment. Also, the old 'vehicle centred' notion in traffic engineering will be replaced by the new 'human centred' notion which takes all road users into consideration in its planning, design and operations and attaches more importance to vulnerable traffic participants in this case the pedestrians.

\section{CONCLUSION}

The safety of pedestrians crossing at traffic warden controlled intersections can be enhanced through the introduction of an all-stop exclusive pedestrian phase into the traffic management scheme at TWC multilane intersections with high volumes of pedestrians and vehicular traffics and high vehicular speeds. This will eliminate the often noticed nervous anxiety seen in pedestrians as they attempt to cross at such intersections. Such a scheme will require the introduction of pedestrian crosswalk. The crosswalk is necessary to concentrate pedestrian crossing activity at a specific location and position pedestrians to be more visible by traffic wardens and motorists. The approaches at the intersections should also be provided with stop bars which will serve as a guide to indicate the optimal stopping location for motorists to stop further back from the crosswalk. This recommended traffic warden controlled management method which is both human and vehicle centered will reduce the risk in conflict between vehicular and pedestrian movements and thereby encourage and promote walking which is one of the non-motorised modes of transportation that is currently being encouraged worldwide with the benefits of enhanced health, reduction of vehicular traffic congestions and the emission of greenhouse gasses thus creating a friendly environment.

The modified traffic signal phasing proposed in the study should be introduced at the studied intersection and similar TWC multilane intersections with high vehicular and pedestrian traffic volumes with high traffic speeds so as to enhance pedestrian movements. It will be a good scheme in making a community more walkable. 


\section{REFERENCES}

[1] Adeleke, 0.0. and Jimoh, Y.A. "Sampling of Intersections for Traffic Delay Study in an African Sub Region Urban City", Epistemics in Science, Engineering and Technology, Vol. 1, No. 3, 2011, pp 131-137.

[2] Garber, N. J. and Hoel, L. A. Traffic and Highway Engineering, Cengage Learning, 2010.

[3] Hamidun, R., Ishak, S. T. and Endut, I. R. 'Assessing Pedestrian Crossing Risk at Signalised Intersection', International Journal of Emerging Technology and Advanced Engineering, Vol. 3, No. 1, 2013,pp 31 - 35.

[4] US Dept of Admin. 'Pedestrian Safety at Intersections', Federal Highway Administration, 2009.

[5] Alhajyaseen, W. K. M., Asano, M. and Nakamura, H. 'Estimation of Left-turning Vehicle, Maneuvers for the Assessment of Pedestrian Safety at Intersections', Journal International Association of Traffic and Safety Sciences, IATSS Research 36, 2012, pp 66-74.

[6] Brisbane City Council."Roads and Traffic Sheet Traffic Lights", 2008

http://www.brisbane.qld.gov.au/documents/service s/traffic\%20signals.pdf Accessed on April 11, 2013.

[7] Gupta, D. and Patel, V. R. 'Simulation of Pedestrian at Intersection in Urban Congested Area', International Journal of Research in Engineering and Technology, Vol. 3, No. 5, 2014, pp $40-44$.

[8] Lukeman, Y. Gbadamosi, T.K. and Omole, F. K. 'Analysis of Walking and Trekking as an Alternative to Motorised Trip in Akoka, Lagos, Nigeria', International Journal of Education and Research, Vol. 3, No. 1, 2015,pp 247 -258.

[9] Mwakalonge, J. L., Waller, J. C., and Perkins, J. A. 'Temporal Stability and Transferability of NonMotorized and Total Trip Generation Models',
Journal of Transportation Technologies, 2012.http://www.SciRP.org/journal/itts Accessed on September 21, 2015.

[10] Jain, A., Gupta, A. and Rastogi, R. 'Pedestrian Crossing Behaviour Analysis at Intersections' International Journal for Traffic and Transport Engineering, Vol. 4, No. 1, 2014, pp 103 - 116.

[11] Pollack K. M., Gielen, A. C., Ismail, M. N. M., Mitzner, M., $\mathrm{Wu}, \mathrm{M}$. and Links, J. M. Investigating and Improving Pedestrian Safety in an Urban Environment', Injury Epidemiology, Vol. 1, No. 1, 2014, pp 1 - 9.

[12] Department for Regional Development, Northern Ireland. Traffic Signs Manual, Chapter 5 Road Markings, Department for Regional Development (Northern Ireland) Scottish Executive, Welsh, Assembly Government, London, 2009.

[13] United States Department of Transportation. Manual on Uniform Traffic Control Devices, Millennium Edition, Federal Highway Administration 2000.

[14] Garder, P. 'Pedestrian Safety at Traffic Signals: A Study Carried Out With the Help of a Traffic Conflicts Technique', Acod. and B Pm., Vol. 21, No. 5, 1989, pp. 435-444.

[15] Adeleke, O. O., Jimoh, Y. A., Salami, A. W. "Determination of Capacity at Traffic Warden Controlled Intersection Using Fixed-Time Signalised Intersection Capacity Model". International Journal of Engineering, Tome XI, Fascicule 3, 2013, pp. 33 36.

[16] O'Flaherty C. A. Transport Planning and Traffic Engineering, Elsevier Ltd., UK, 1997.

[17] Institute of Transportation Engineers. Design and Safety of Pedestrian Facilities, A Recommended Practice, Institute of Transportation Engineers, 1998. 